\title{
Efecto hepatoprotector del extracto acuoso de Smallanthus sonchifolius (yacón) en un modelo de intoxicación con acetaminofén
}

Hepatoprotective effect of Smallanthus sonchifolius (yacon) aqueous extract in an acetaminophen intoxication model

\author{
Acela Inés Arnao-Salas ${ }^{1}$, Silvia Suárez-Cunza ${ }^{1}$, Juan Trabucco-Ricaldi ${ }^{2}$, \\ Ruth Cisneros-Chinchay ${ }^{2}$, María Elena-Rodrigo ${ }^{2}$ \\ Centro de Investigación de Bioquímica y Nutrición “Alberto Guzmán Barrón”, Facultad de Medicina, Universidad Nacional Mayor de San \\ Marcos, Lima, Perú. \\ ${ }^{2}$ Maestría en Bioquímica, Unidad de Posgrado, Facultad de Medicina, Universidad Nacional Mayor de San Marcos, Lima, Perú.
}

\begin{abstract}
Resumen
En la medicina tradicional se ha publicado que las hojas de Smallanthus sonchifolius (yacón) poseen efectos antidiabético y hepatoprotector. Objetivos: Evaluar en suero y hematies el efecto hepatoprotector del extracto acuoso de hojas de yacón (EAY) en un modelo de intoxicación con acetaminofén en ratas. Diseño: Experimental, transversal. Institución: Centro de Investigación de Bioquímica y Nutrición, Facultad de Medicina, Universidad Nacional Mayor de San Marcos, Lima, Perú. Material biológico: Hojas de yacón. Intervenciones: Se formó cinco grupos de ratas hembra $(n=6)$ que recibieron por cinco días, por vía oral, suero fisiológico (SF), EAY o silimarina (Sil) $(50 \mathrm{mg} / \mathrm{kg}$ ) y luego de 1 hora, SF o acetaminofén (A) $250 \mathrm{mg} / \mathrm{kg}$, según lo siguiente: G1 (control; SF-SF), G2 (SF-A), G3 (EAY (200 mg/kg)-A), G4 (EAY (400 mg/kg)-A) y G5 (Sil-A). Principales medidas de los resultados: Actividad de aspartato amino transferasas (AST), alanina amino transferasa (ALT), fosfatasa alcalina (FAL) y $\gamma$-glutamiltranspeptidasa $(\gamma$-GTP); niveles de bilirrubina total (BT), proteinas y lipoperoxidación (MDA). En hematies, actividades de superóxido dismutasa (SOD), catalasa (CAT) y hemoglobina. Resultados: Se observó aumento significativo $(p<0,05)$ en la actividad de $\gamma$-GTP entre el grupo $G 2$ y los grupos $G 3$ y G4. Hubo disminución significativa $(p<0,05)$ de proteínas en el grupo $G 2$ con respecto $G 1$. El nivel de MDA fue menor en el grupo que recibió $200 \mathrm{mg} / \mathrm{kg}$ de EAY con respecto al control. Las actividades de AST, ALT y FAL no mostraron diferencias significativas. La relación SOD/CAT fue similar entre los grupos $\mathrm{G} 1, \mathrm{G} 4$ y G5, evidencia de una recuperación del daño causado por el acetaminofén. Conclusiones: La administración del EAY tuvo un efecto hepatoprotector comparable a la silimarina.
\end{abstract}

Palabras clave: Smallathus sonchifolius, plantas medicinales, extractos vegetales, marcadores biológicos, catalasa, superóxido dismutasa, acetaminofeno.

Abstract

In traditional medicine Smallanthus sonchifolius (yacon) leaves are reported to have antidiabetic and hepatoprotective effects. Objectives: To determine in serum and erythrocytes the yacon leaves (EAY) aqueous extract hepatoprotective effect in a model of acetaminophen poisoning in rats. Design: Experimental, cross sectional. Institution: Biochemistry and Nutrition Research Center, Faculty of Medicine, Universidad Nacional Mayor de San Marcos, Lima, Peru. Biological material: Yacon leaves. Interventions: Five groups of female rats $(n=6)$ received orally for five days either saline (SF), EAY or silymarin (Sil) $(50 \mathrm{mg} / \mathrm{kg})$ and after 1 hour, SF or acetaminophen (A) $250 \mathrm{mg} / \mathrm{kg}$, as follows: G1 (control; SF-SF), G2 (SF-A), G3 (EAY (200 mg/kg)-A), G4 (EAY (400 mg/kg)-A) and G5 (Sil-A). Main outcome measures: Aspartate amino transferase activity (AST), alanine amino transferase (ALT), alkaline phosphatase (ALP), and $\gamma$-glutamyltranspeptidase ( $\gamma$-GTP); total bilirubin levels (BT), protein and lipid peroxidation (MDA). Superoxide dismutase (SOD), catalase (CAT) and hemoglobin activities in erythrocytes. Results: There was significant increase of $\gamma$-GTP activity $(p<0.05)$ between group $G 2$ and groups $G 3$ and $G 4$, and significant decrease $(p<0.05)$ in proteins in group $G 2$ in relation to group $G 1$. MDA level was lower in the group receiving $200 \mathrm{mg} / \mathrm{kg}$ of EAY with respect to control. AST, ALT and FAL activities showed no significant differences. SOD/CAT ratio was similar between groups $\mathrm{G} 1, \mathrm{G} 4$ and $\mathrm{G} 5$, evidence of recovery from acetaminophen damage. Conclusions: EAY administration had hepatoprotective effect comparable to silymarin.

Key words: Smallanthus sonchifolius, plants/medicinal, plant extracts, biological markers, catalase, superoxide dismutase, acetaminophen.

An Fac med. 2012;73(3):239-44

\section{INTRODUCCIÓN}

En los últimos años ha crecido el interés por el estudio de plantas con propiedades medicinales para demostrar sus efectos farmacológicos. Miranda ML y colaboradores comunicaron que el uso de la medicina alternativa complementaria en las enfermedades hepá- ticas en Europa es del 20 al 65\%, en Estados Unidos, 39\% y, en México, 85\%, siendo las mujeres mayores de 40 años las principales usuarias ${ }^{(1)}$.

En el Perú, la cirrosis hepática ocupa el quinto lugar como causa de mortalidad y segundo como morbilidad, siendo consecuencia de la intoxicación con alcohol, infecciones virales, cáncer, fármacos, entre otros ${ }^{(2)}$. El estudio de hepatopatías en modelos animales se realiza mediante la administración crónica con tetracloruro de carbono ${ }^{(3,4)}$, acetaminofén ${ }^{(5,6)}$ y etanol.

El acetaminofén (paracetamol) es un medicamento hepatotóxico de ven- 
ta libre. Su detoxificación hepática es principalmente por fase II, conjugado con el ácido glucurónico y sulfato; una parte minoritaria se oxida en fase I, mediante el CYP2E1, y forma un compuesto electrofílico, el NAPQI ( $\mathrm{N}$ acetil benzoquinonemina), que reacciona con el glutatión.

El estrés oxidativo -desbalance entre los agentes prooxidantes y antioxidantes- está asociado a diversos procesos fisiológicos y patológicos. Puede evitarse disminuyendo las reacciones prooxidantes que generan especies reactivas de oxígeno (EROs) o incrementando los componentes antioxidantes que pueden ser consumidos mediante alimentos y extractos de plantas.

Un estudio reciente realizado por Adewusi y Afolayan informa de 107 plantas usadas en el tratamiento de enfermedades hepáticas y concluyen que son una nueva alternativa ${ }^{(7)}$. La fitoterapia emplea plantas tales como boldo (Peumus boldus), verbena (Verbena carolina L), cardo mariano (Silybum marianum), hercampuri (Gentianella alborosea), alcachofa (Cynara scolymus), perejil (Petroselinum sativum), Alchornea cordifolia, entre las más conocidas ${ }^{(1,4-6)}$.

Los efectos hepatoprotectores de las preparaciones herbales se deben a su contenido en compuestos polihidroxilados: fenoles, flavonoides, terpenoides y otros metabolitos secundarios ${ }^{(1)}$ con actividad antioxidante, que pueden convertir a las EROs en productos más estables. Una planta nativa con alto contenido de antioxidantes es el yacón.

Smallanthus sonchifolius es conocido como yacón, llacón, aricoma, jícama, llaqon, llacum, llacuma, yacumpi, aricuma, chicama, jiquima y jiquimilla. En la medicina tradicional peruana se usa las hojas y la raíz reservante de yacón. Es empleado para rejuvenecer la piel y aliviar problemas gastrointestinales, hepáticos y renales ${ }^{(8)}$. Estudios in vitro realizados en nuestro laboratorio han demostrado que el extracto acuoso de hojas de yacón es una rica fuente de antioxidantes ${ }^{(9)}$. Es poco documentado

Tabla 1. Tratamiento experimental del EAY en un modelo de intoxicación con acetaminofén.

\begin{tabular}{|c|c|c|c|c|}
\hline \multicolumn{1}{|c|}{ G1 control } & G3 & G4 & G5 \\
\hline Suero fisiológico & Suero fisiológico & EAY $200 \mathrm{mg} / \mathrm{kg}$ & EAY $400 \mathrm{mg} / \mathrm{kg}$ & Silimarina $50 \mathrm{mg} / \mathrm{kg}$ \\
\hline \multicolumn{5}{|c|}{ Después de una hora } \\
\hline Suero fisiológico & $\begin{array}{c}\text { Acetaminofén } \\
250 \mathrm{mg} / \mathrm{kg}\end{array}$ & $\begin{array}{c}\text { Acetaminofén } \\
250 \mathrm{mg} / \mathrm{kg}\end{array}$ & $\begin{array}{c}\text { Acetaminofén } \\
250 \mathrm{mg} / \mathrm{kg}\end{array}$ & $\begin{array}{c}\text { Acetaminofén } \\
250 \mathrm{mg} / \mathrm{kg}\end{array}$ \\
\hline \multicolumn{5}{|c|}{ Después de una hora: realimentación } \\
\hline
\end{tabular}

el efecto hepatoprotector de las hojas de yacón. De allí nuestro interés en realizar la presente investigación para evaluar su acción farmacológica en un modelo de intoxicación hepática con acetaminofén.

\section{METODOLOGÍA}

El acetaminofén y la silimarina fueron obtenidos de la casa SIGMA Co. Los reactivos fueron de grado analítico y para el perfil hepático se usó kits comerciales de la casa Wienner.

Se utilizó las hojas de yacón de la variedad Blanco Ecuatoriano, cultivadas en el Campo Experimental del Programa de Raíces y Tubérculos Andinos de la Universidad Nacional de Cajamarca (2 $536 \mathrm{msnm}$ ). La variedad corresponde a Smallanthus sonchifolius (Poepp. \& Endl.) H. Robinson, según el reporte del Museo de Historia Natural de la UNMSM. Las hojas fueron lavadas, secadas y molidas.

Se preparó una infusión al 2\% (p/v) en agua hirviendo, se filtró en frío usando papel Wahtman №1; se preparó alícuotas que fueron colocadas en la estufa a $37^{\circ} \mathrm{C}$, hasta obtener el extracto seco. Se mantuvo en congelación a $-20^{\circ} \mathrm{C}$ hasta antes de su uso.

La determinación de la propiedad antioxidante se realizó mediante la captación del radical 2,2-difenil-1picrilhidrazilo (DPPH). Se cuantificó los polifenoles y flavonoides empleando ácido gálico y quercetina como estándares, según los métodos previamente publicados ${ }^{(9)}$.
Para la evaluación del efecto hepatoprotector, se usó ratas hembras adultas, raza Holtzman, con un peso promedio de 220 a $240 \mathrm{~g}$, procedentes del bioterio de la Universidad Nacional Agraria La Molina (UNALM). Los animales se aclimataron una semana y se les pesó cada dos días. Se les alimentó con dieta obtenida de UNALM y agua ad líbitum, en condiciones ambientales estandarizadas. Luego de dicho período, se procedió a formar aleatoriamente los cinco grupos de estudio $(n=6)$. Antes del tratamiento con el extracto acuoso de yacón (EAY), se les retiró el alimento y recibieron por vía orogástrica durante cinco días lo señalado en la tabla 1.

Los animales fueron sacrificados 20 horas después de la última administración, siguiendo las recomendaciones éticas establecidas para el cuidado de animales de laboratorio. Se obtuvo muestras de sangre total en tubos heparinizados y de suero para los análisis bioquímicos siguientes:

El perfil hepático se evaluó mediante las enzimas séricas, transaminasas (aspartato aminotransferasa, AST, y alanina aminotransferasa, ALT), según el método de Reitman S y Frankel F ${ }^{(10)}$; fosfatasa alcalina (FAL) mediante el método propuesto por Bowers GN y McComb RB ${ }^{(11)}$, y $\gamma$-glutamiltranspeptidasa $\left(\gamma\right.$-GTP) según Szasz $\mathrm{G}^{(12)}$. Otros parámetros ensayados fueron la bilirrubina total (BT) ${ }^{(13)}$ y proteínas (PRT) ${ }^{(14)}$.

Los niveles de malondialdehido (MDA), indicador de lipoperoxidación, fueron determinados según Buege y Aust ${ }^{(15)}$. Para distinguir la enzima superóxido dismutasa (SOD, EC 1.5.1.1) 
Tabla 2. Efecto del extracto acuoso de yacón en los biomarcadores séricos de daño hepáticos en ratas intoxicadas con acetaminofén.

\begin{tabular}{ccllllll} 
Grupos & AST (U/L) & ALT (U/L) & FAL (U/L) & $\gamma-$ GTP (U/L) & PRT (g/L) & BT (mg/dL) & MDA (nmol/mL) \\
G1 & $64,4 \pm 26,8$ & $35,3 \pm 10,4$ & $284,8 \pm 109,9$ & $15,3 \pm 7,3^{\mathrm{a}, \mathrm{b}}$ & $7,6 \pm 0,3^{\mathrm{a}}$ & $0,09 \pm 0,01^{\mathrm{a}}$ & $7,5 \pm 1,8$ \\
G2 & $66,2 \pm 15,5$ & $37,8 \pm 9,2$ & $270,2 \pm 83,9$ & $24,2 \pm 6,8$ & $7,0 \pm 0,4^{\mathrm{a}}$ & $0,11 \pm 0,01^{\mathrm{a}, \mathrm{b}}$ & $5,7 \pm 1,0^{\mathrm{a}, \mathrm{b}}$ \\
G3 & $54,7 \pm 7,4$ & $40,6 \pm 5,3$ & $223,3 \pm 99,3$ & $17,8 \pm 6,5^{\mathrm{c}, \mathrm{d}}$ & $7,2 \pm 0,4$ & $0,09 \pm 0,02$ & $6,0 \pm 1,2^{\mathrm{c}}$ \\
G4 & $57,6 \pm 17,4$ & $37,2 \pm 9,2$ & $230,8 \pm 65,9$ & $26,5 \pm 3,4^{\mathrm{a}, \mathrm{c}}$ & $7,5 \pm 0,5$ & $0,11 \pm 0,02$ & $7,7 \pm 1,0^{\mathrm{a}}$ \\
G5 & $58,8 \pm 11,1$ & $35,0 \pm 7,4$ & $306,0 \pm 116,3$ & $28,7 \pm 6,7^{\mathrm{a}, \mathrm{b}, \mathrm{d}}$ & $7,2 \pm 0,5$ & $0,09 \pm 0,0^{\mathrm{b}}$ & $7,6 \pm 1,3^{\mathrm{b}, \mathrm{c}}$ \\
\hline
\end{tabular}

${ }^{*}$ En las columnas, letras iguales tienen significancia $(p<0,05)$.

se empleó el método de Marklund y Marklund (16) y para la catalasa (CAT, EC 1.11.1.6), el método según Aebi ${ }^{(17)}$.

Una unidad de SOD (U) fue definida como la cantidad de enzima necesaria para reducir en 50\% la autooxidación del pirogalol a $\mathrm{pH}$ alcalino. La actividad se expresó en U SOD/g hemoglobina.

Una unidad de catalasa (U) equivale a la cantidad de enzima que cataliza la descomposición de un micromol de peróxido de hidrógeno por minuto, bajo las condiciones de ensayo. Se usó el coeficiente de extinción $\varepsilon 240=39,4 \mathrm{M}^{-1}$. $\mathrm{cm}^{-1}$. La actividad fue expresada en $\mathrm{U}$ CAT/g hemoglobina.

Los resultados séricos son informados como el promedio + su desviación estándar y fueron analizados empleando la prueba t-student; las diferencias han sido consideradas significativas cuando $p<0,05$. Se realizó un análisis descriptivo para el análisis de los datos de las actividades enzimáticas de SOD y catalasa y los resultados son presentados como medianas.

\section{RESULTADOS}

La capacidad antioxidante medida en base al contenido de fenoles totales y flavonoides del extracto acuoso de yacón fue de $736,3 \pm 18,4 \mathrm{ug} / \mathrm{mL}$ y 134,9 $\pm 8,8 \mathrm{ug} / \mathrm{mL}$, respectivamente. Además el EAY demostró una fuerte capacidad antioxidante $\left(\mathrm{IC}_{50}=25,97 \pm 1,03 \mathrm{ug} /\right.$ $\mathrm{mL}$ ) frente al radical libre DPPH.

Las determinaciones de los marcadores séricos empleados para evaluar el daño hepático producido tras la intoxicación con acetaminofén en las ratas, se muestra en la tabla 2.

En la tabla anterior podemos observar que la actividad de $\gamma$-GGT es la que muestra diferencias significativas. El grupo que recibió acetaminofén mostró una disminución significativa frente al control. Los valores de bilirrubina total variaron entre 0,09 y $0,11 \mathrm{mg} / \mathrm{dL}$; el incremento significativo se produjo en el grupo que sufrió la injuria. La formación de malondialdehído disminuyó significativamente en los grupos G2 y G3.

Por otro lado, las actividades de las enzimas SOD y catalasa en los eritrocitos, informadas en medianas, son mostradas en las gráficas 1 y 2 , respectivamente

Las actividades de la SOD se vieron incrementadas en el grupo G2 y disminuidas en los grupos G4 y G5, con respecto al control. El $\mathrm{H}_{2} \mathrm{O}_{2}$ generado por esta enzima fue neutralizado por la glutatión peroxidasa y catalasa. Si observamos la gráfica 2, vemos que todos los valores de catalasa en los grupos experimentales fueron menores con respecto al G1; y en el caso de G2, que

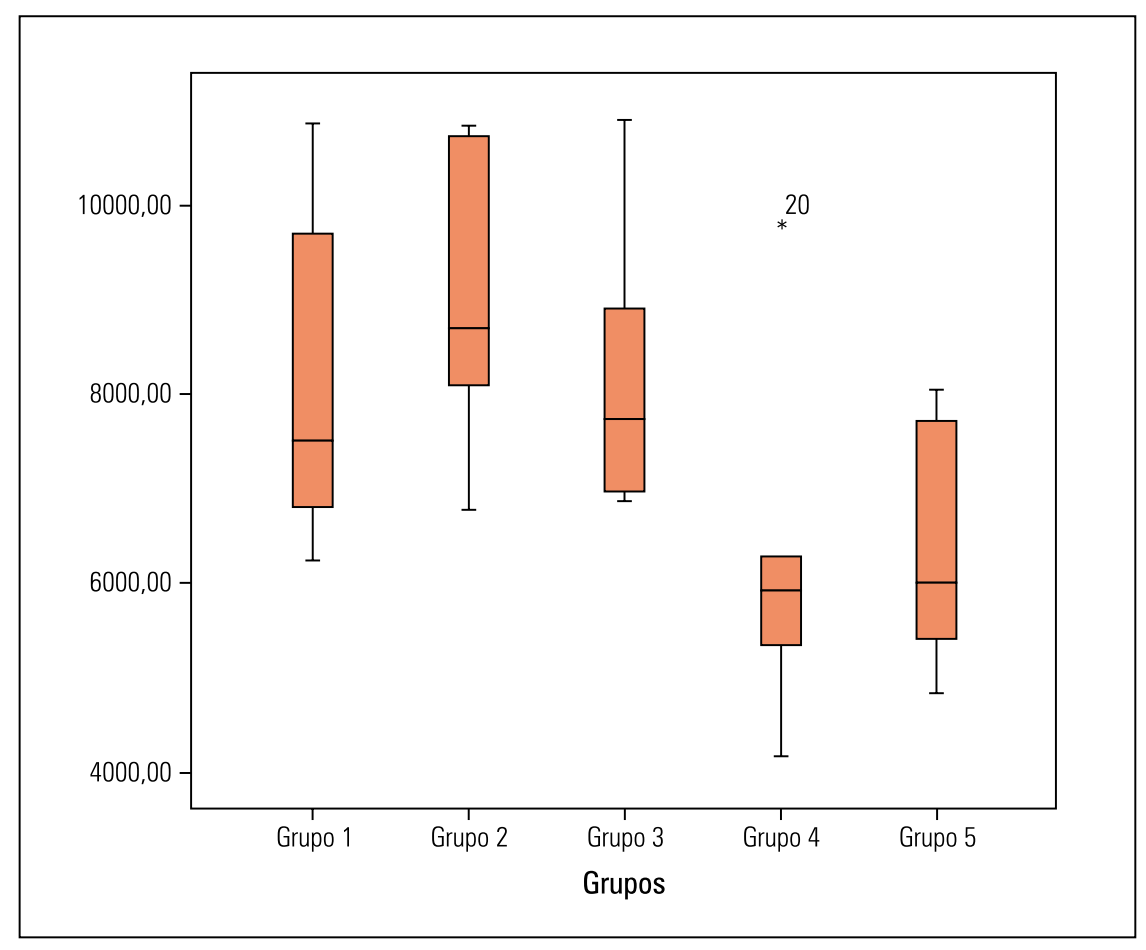

Gráfica 1. Evaluación del efecto del EAY en la actividad de SOD en glóbulos rojos. 
recibió tratamiento con paracetamol, estuvo fuertemente reprimida; estos comportamientos influyeron en las variaciones de la relación SOD/CAT que se muestra en la gráfica 3 , en el que se observa un valor aumentado en los grupos $\mathrm{G} 2$ y $\mathrm{G} 3$.

\section{DISCUSIÓN}

Basándonos en estudios previos que comunican un alto contenido de antioxidantes en las hojas de yacón ${ }^{(9)}$, ensayamos su acción como agente hepatoprotector en una intoxicación inducida con acetaminofén en ratas. Dicho extracto presentó buena capacidad antioxidante, según los indicadores que evaluamos, siendo su $\mathrm{IC}_{50} 25,97 \mathrm{ug} /$ $\mathrm{mL}$, lo que estuvo dentro de lo encontrado por Valentová para los extractos de hojas de yacón $\left(\mathrm{IC}_{50}\right.$ 16,14 a 33,39 $\mathrm{ug} / \mathrm{mL})^{(18)}$. El contenido de polifenoles totales $(736 \mathrm{ug} / \mathrm{mL})$ fue mayor al de otras especies, como A. cordifolia (220 $\mathrm{ug} / \mathrm{mL}$ ), que ha demostrado tener un efecto hepatoprotector ${ }^{(6)}$.

Los polifenoles y flavonoides están presentes en frutas, verduras, extractos vegetales, y constituyen una excelente fuente de antioxidantes que pueden contribuir a restablecer el equilibrio prooxidante/antioxidante en una situación de estrés oxidativo.

Es necesario mantener el equilibrio prooxidante/antioxidante en los organismos aerobios, porque están expuestos a la generación endógena de EROs, tales como el anión superóxido $\left(\mathrm{O}_{2}^{-}\right)$, el peróxido de hidrógeno $\left(\mathrm{H}_{2} \mathrm{O}_{2}\right)$, el radical hidroxilo $\left(\mathrm{OH}^{-}\right)$, el óxido nítrico (NO), peróxidos $\left(\mathrm{ROO}^{-}\right)$, los cuales dañan las diversas estructuras celulares. En esta situación es muy importante la acción de sustancias endógenas y exógenas que neutralizan dichos efectos nocivos de las EROs.

El hígado es el encargado de los procesos de detoxificación en fase I y fase II. En una intoxicación con acetaminofén, su catabolismo principal por la fase II cambia a la fase I produciendo

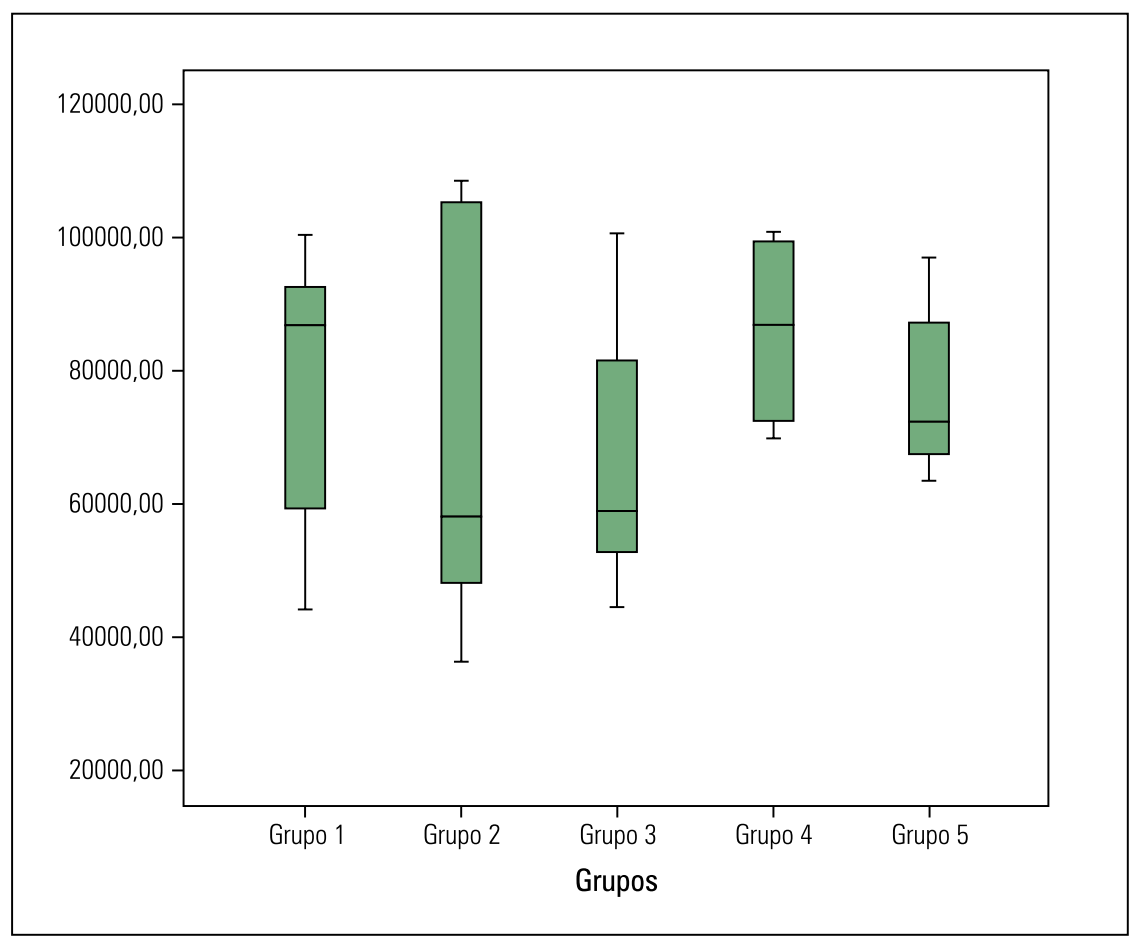

Gráfica 2. Evaluación del efecto del EAY en la actividad de catalasa en glóbulos rojos. el radical NAPQI, que ocasiona daños en la arquitectura celular; al agotar la reserva de GSH y por la propagación de las EROS que no llegan a ser neutralizadas por los mecanismos antioxidantes, se establece una situación de estrés oxidativo y daño hepatocelular.
El daño generado fue evaluado midiendo las actividades de las enzimas plasmáticas: transaminasas, fosfatasa alcalina y $\gamma$ glutamil transpeptidasa.

Las actividades de las AST y ALT no evidenciaron un incremento significativo entre los grupos experimentales

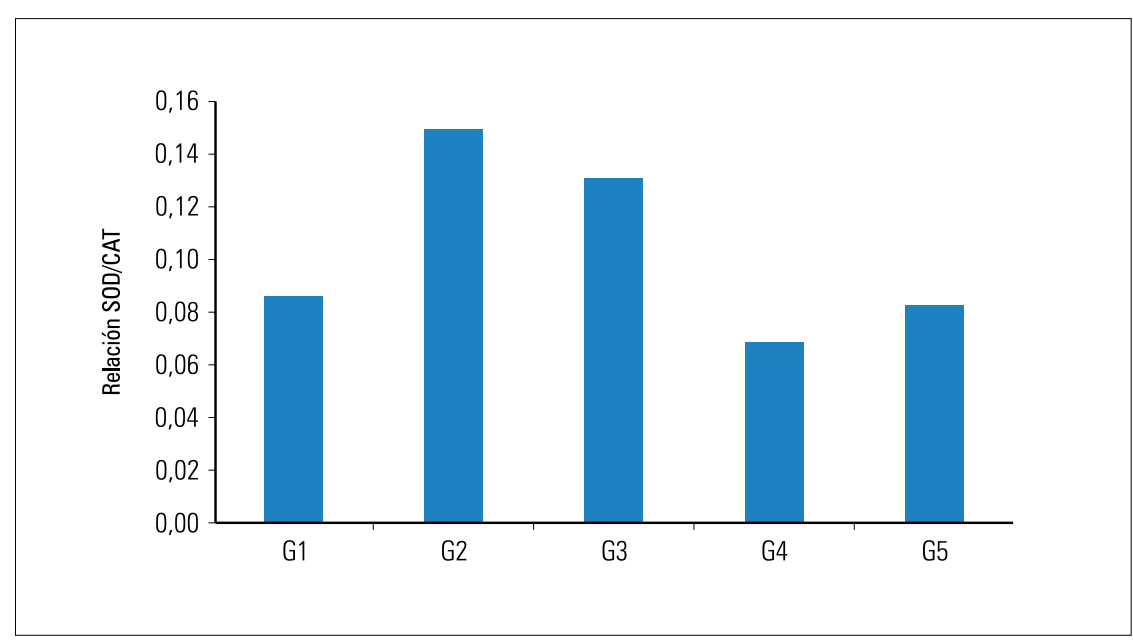

Gráfica 3. Evaluación del efecto del EAY en la relación de actividad de SOD/actividad de catalasa en los glóbulos rojos. 
y esto concuerda con lo encontrado por Troncoso y Guija ${ }^{(5)}$, quienes evaluaron el efecto hepatoprotector del perejil. Ellos encontraron valores menores para la AST y ALT en el grupo tratado con acetaminofén más perejil; sin embargo, nosotros lo observamos solo para la AST.

En las otras dos enzimas -fosfatasa alcalina y $\gamma$ glutamil transpeptidasa- se observó cambios significativos para esta última, en los grupos con acetaminofén, EAY a $400 \mathrm{mg} / \mathrm{kg}$ y silimarina (hepatoprotector), al ser comparados con el control. Troncoso y Guija encontraron diferencias significativas en todos los grupos y, para el caso del perejil, el valor de la $\gamma$ GTP fue comparable al control, así como también lo encontramos para el grupo que recibió EAY a $200 \mathrm{mg} / \mathrm{kg}$. Esta última enzima es un marcador muy sensible a la acción de las EROs, pues está ligada a la membrana; y el daño a nivel hepático en intoxicaciones agudas se manifiesta con aumentos significativos de su actividad plasmática.

A diferencia de los incrementos leves que produce la intoxicación con acetaminofén, se ha informado incrementos de hasta tres veces de la actividad con la ALT ${ }^{(4)}$ cuando se usó el $\mathrm{Cl}_{4} \mathrm{C}$; este produce un daño más agresivo, probablemente con necrosis celular. Valores incrementados de ALT también se obtiene cuando se trabaja con la fracción post mitocondrial de hígado ${ }^{(3)}$. Así mismo, se elevan dramáticamente las actividades de la FAL en un 100\% y para la $\gamma$ GTP en $500 \%$ con respecto al control (4).

En su mecanismo de detoxificación, el acetaminofén puede sobrepasar las barreras antioxidantes y formar especies reactivas que tienen como su principal blanco los lípidos de las membranas celulares. Una manera de evaluar este daño es mediante la prueba de lipoperoxidación.

La formación de MDA-TBARS fue semejante entre el control y los grupos G4 y G5, pero los que recibieron paracetamol y EAY a $200 \mathrm{mg} / \mathrm{kg}$ mostraron valores menores; esto podría ser explicado porque la dosis empleada y los mecanismos antioxidantes a nivel hepático todavía estaban controlando dicho estrés.

Otro biomarcador no enzimático medido fue la bilirrubina total, que estuvo significativamente elevada en el grupo con paracetamol y silimarina, pero no en los que recibieron tratamiento con el EAY. Esto difiere de lo encontrado para A. cordifolia, que requirió de una dosis del extracto de $500 \mathrm{mg} / \mathrm{kg}$ de peso para restablecer los valores normales de bilirrubina total. En todo caso, cuando se usa intoxicación con $\mathrm{Cl}_{4} \mathrm{C}$, los incrementos en la bilirrubina son hasta de tres veces.

Finalmente, las proteínas tuvieron menores valores en el grupo con paracetamol, siendo posible que este recambio permita una mayor disponibilidad de aminoácidos para la síntesis de proteínas o péptidos con propiedades antioxidantes. En el estudio realizado por Troncoso y Guija no se encontró variación en los valores de proteínas, albúminas y glutatión a nivel sérico, aunque los niveles de los últimos parámetros (albúmina y glutatión) fueron menores en el grupo que recibió perejil con respecto a los otros grupos, sin ser significativo.

Resumiendo los datos obtenidos hasta aquí, podemos decir que la intoxicación con acetaminofén a la dosis de $250 \mathrm{mg} / \mathrm{kg}$ en ratas hembras, evidenciada por las actividades aumentadas de AST, ALT, $\gamma$ GTP $(p<0,05)$, y los niveles incrementados de bilirrubina total y disminuidos de proteínas totales $(p<0,05)$ fue atenuada por la administración de EAY.

El efecto de la dosis de EAY en los diferentes biomarcadores a nivel sérico usados en el presente estudio, encontramos que a la dosis de $200 \mathrm{mg} / \mathrm{kg}$ de peso es un mejor hepatoprotector, porque se obtiene valores menores de AST, fosfatasa alcalina, $\gamma$ glutamil transpeptidasa y bilirrubina total, con respecto al grupo de ratas intoxicado con acetaminofén. En el caso de la dosis de EAY a $400 \mathrm{mg} / \mathrm{kg}$, los valores fueron menores en AST y bilirrubina total, con respecto al grupo que recibió acetaminofén. Sin embargo, la dosis efectiva EAY podría ser confirmada mediante estudios histopatológicos a nivel hepático.

Olaleye y colaboradores ${ }^{(6)}$ demostraron el efecto hepatoprotector de A. cordifolia en un modelo de intoxicación con acetaminofén en ratas machos, usando una dosis mucho mayor $(2 \mathrm{~g} /$ $\mathrm{kg}$ ), lo que produjo disminución significativa de las actividades de AST, ALT y fosfatasa alcalina. En nuestro caso, la disminución fue significativa solo con la actividad de la $\gamma$ glutamiltranspeptidasa y nuestros resultados son comparables a los de Troncoso y Guija, quienes demostraron el efecto hepatoprotector del perejil usando una dosis de $200 \mathrm{mg} /$ $\mathrm{kg}$ de acetaminofén en ratas machos.

Es conocido que los eritrocitos están expuestos a una alta tensión de oxígeno y en una situación de estrés oxidativo el daño producido a las macromoléculas es irreparable. Neutralizan tal situación mediante sus sistemas protectores, que incluyen a la SOD, catalasa, glutatión reducido, glutatión peróxidasa, glutatión $\mathrm{S}$ transferasa y glutatión reductasa. Estas bloquean la reacción en cadena originada por los radicales libres.

Para valorar los mecanismos antioxidantes en el eritrocito medimos las actividades de SOD y catalasa y encontramos que los valores de SOD están ligeramente incrementados en el grupo que recibió acetaminofén (gráfica 1). En el eritrocito encontramos la isoenzima SOD Cu-Zn, que actúa produciendo la dismutación del $\mathrm{O}_{2}$ y formando peróxido de hidrógeno $\left(\mathrm{H}_{2} \mathrm{O}_{2}\right)$. Este es un agente nocivo con propiedades prooxidantes y es sustrato de la catalasa y la glutatión peroxidasa.

La catalasa es una metaloproteína tetramérica que se encuentra en el citosol del eritrocito y participa descomponiendo el $\mathrm{H}_{2} \mathrm{O}_{2}$ en $\mathrm{H}_{2} \mathrm{O}$ y $1 / 2 \mathrm{O}_{2}$ Estas dos enzimas trabajan en forma asociativa, para evitar la formación del radical más dañino, el $\mathrm{OH}$. 
En nuestro caso, la actividad de la catalasa estuvo más baja en el G2 y la administración de EAY o silimarina preservaron su actividad, si se compara a los del control (gráfica 2). La actividad incrementada de SOD y disminuida de catalasa hace que la relación de las medianas de SOD/CAT (gráfica 3) aumente si se compara con el grupo G1. Este valor se interpreta como un mayor daño al glóbulo rojo, debido a que la actividad de catalasa no responde a la mayor producción de superóxido, generando una protección baja contra los efectos oxidativos del $\mathrm{H}_{2} \mathrm{O}_{2}$. Esta relación empieza a disminuir en el grupo G3 y es más evidente en el grupo G4, comparándose el resultado con el grupo G5 que fue tratado con el hepatoprotector estándar. El EAY muestra entonces su papel hepatoprotector a nivel del glóbulo rojo.

Nuestro equipo de investigación también ha estudiado los sistemas antioxidantes a nivel hepático, lo que aunado al presente estudio nos dará una visión más integral de los mecanismos antioxidantes que participan en la acción hepatoprotectora de las hojas de yacón.

En conclusión, de acuerdo al modelo experimental de intoxicación con acetaminofén en ratas, el extracto acuoso de hojas de yacón presentó una acción hepatoprotectora que se correlacionó con su capacidad antioxidante y fue comparable a la silimarina.

\section{AGRADECIMIENTOS}

Los autores agradecen al VRI-CSI de la UNMSM por el apoyo a la presente investigación (Código 100104291).

Un agradecimiento especial al Ing Juan Seminario por habernos proporcionado el material biológico y al QF Rubén Valdivieso por el análisis estadístico de los datos.

Asimismo, los autores agradecen a SECOMAT (Facultad de Ciencias Matemáticas - UNMSM) por el apoyo parcial en la evaluación estadística.

\section{REFERENCIAS BIBLIOGRÁFICAS}

1. Miranda ML, Huacuja L, López AL, Panduro A. Fitoterapia molecular como parte de la medicina alternativa complementaria en las enfermedades del hígado. Investigación en salud, publicación del Centro Universitario de investigación en salud. 2005, VIII: 64-70.

2. Bustíos C, Dávalos M, Román, R, Zumaeta E. Características epidemiológicas y clínicas de la cirrosis hepática en la Unidad de Hígado del HNERM EsSalud. Rev Gastroenterol Perú. 2007;27:238-45.

3. Selema de la Morena G, Martínez Pérez J. Efecto hepatoprotector inducido por el flavonoide astilbina frente a un modelo animal tratado con tetracloruro de carbono. Rev Cubana Plant Med. 1999;1(4):36-9.

4. Favari-PeroziL, Nava-Álvarez R, Meléndez Camargo ME. Probable efecto hepatoprotector de la verbena en la hepatitis inducida con tetracloruro de carbono en la rata. Rev Mex Ciencias Farmacéuticas. 2007;38(3):19-25.

5. Troncoso L, Guija E. Efecto antioxidante y hepatoprotector del Petroselinum sativum (perejil) en ratas, con intoxicación hepática inducida por paracetamol. An Fac med. 2007;68(4):333-43.

6. Olaleye M, Adegboye O, Akindahunsi A. Alchornea cordifolia extract protects wistar albino rats against acetaminophen-induced liver damage. African J Biotechnol. 2006;5(24):2439-45.

7. Adewusi E, Afolayan A. A review of natural products with hepatoprotective activity. J Medicinal Plants Res. 2010;4(13):1318-34.
8. Manrique I, Hermann M, Bernet T. Yacón Ficha técnica. Centro Internacional de la Papa (2004). Disponible en: www.cipotato.org/artc/cip_crops/ fichatecnicayacon.pdf.

9. Arnao I, Seminario J, Cisneros R, Trabucco J. Potencial antioxidante de 10 accesiones de yacón, Smallanthus sonchifolius (Poepp. \&Endl.) H. Robinson, procedentes de Cajamarca-Perú. An Fac med. 2011;72(4):239-43.

10. Frankel S, Reitman S, Sonnenwirth AC, editors. Gradwohl's Clinical Laboratory Methods and

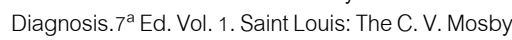
Company; 1970

11. Bowers GN, McComb RB. Measurement of total alkaline phosphatase activity in human serum. Clin Chem. 1975;21(13):1988-95.

12. Szasz Gabor; Hans Ulrich Bergmeyer. Methods of Enzymatic Analysis. Tomo 2, 2. ${ }^{\text {a }}$ edición. 1976:715-9.

13. Watson D. Analytic methods for bilirubin in blood plasma. Clin Chem. 1961;7/6:603.

14. Wiener lab Group. Vademecum. Reagents for Clinical laboratories. Rosario: Wiener Lab Group; 2000.

15. Buege JA, Aust SD. Microsomal lipid peroxidation. Methods Enzymol. 1978;52:302-10.

16. Marklund S, Marklund G. Involvement of the superoxide anion radical in the autoxidation of pyrogallol and a convenient assay for superoxide dismutase. Eur J Biochem. 1974;47(3):469-74.

17. Aebi H. Catalase in vitro. Methods Enzymol. 1984;105:121-6.

18. Valentová K, Šeršeň F, Ulrichová J. Radical scavenging and anti-lipoperoxidative activities of Smallanthus sonchifolius leaf extracts. J Agric FoodChem. 2005; 53(14):5577-82.

\section{Financiamiento}

VRI-CSI de la UNMSM. Código del proyecto No 100104291.

Conflicto de intereses:

Los autores declaran no tener conflicto de intereses.

\section{Correspondencia:}

Mg. Acela Inés Arnao Salas

C. I. de Bioquímica y Nutrición "Alberto Guzmán Barrón"

Facultad de Medicina Humana, UNMSM

Correo electrónico: inesarnao@hotmail.com 